\title{
edificio social
}

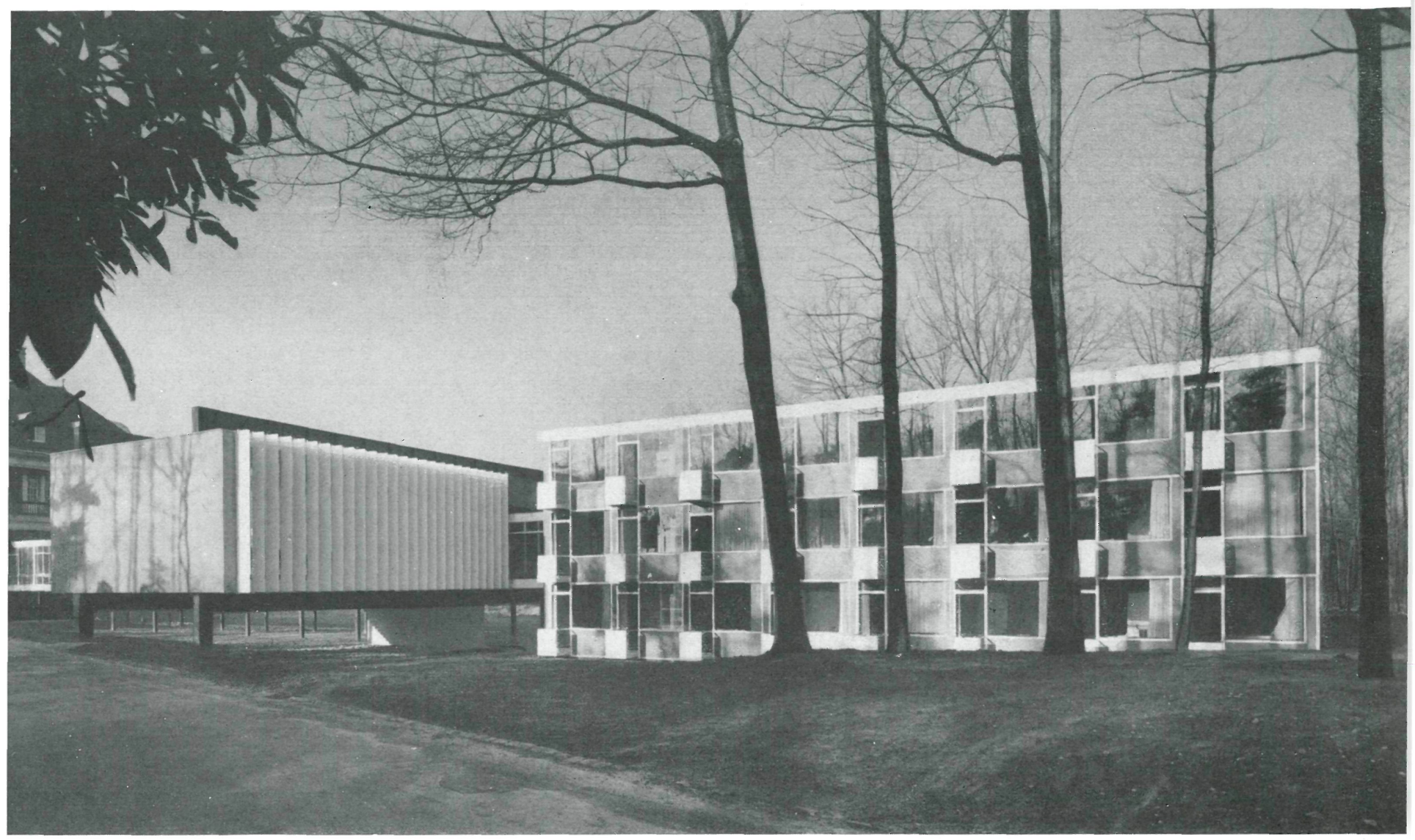

\section{de la academia evangélica en Mirlheim-Ruhr}

B. D. A., arquitecto - F. G. WINTER, ingeniero diplomado

$145-57$

simopsis

La ampliación de las construcciones ya existentes, que se ha llevado a cabo recientemente, comprende: un edificio para huéspedes con 42 habitaciones; un auditorio, y un amplio pasillo de unión del ala nueva con la vieja.

Este pasillo desempeña varias funciones: conecta, en un mismo nivel, la planta principal de la construcción antigua con la sala de conferencias y la planta segunda del edificio residencial; es una galería para pasear y sirve, además, como lugar de relación para establecer contactos personales, entre los congresistas, durante los descansos de conferencia a conferencia.

En el edificio de huéspedes, los forjados de hormigón armado descansan sobre las paredes de separación de las habitaciones.

Respecto a la sala de conferencias, sustentada sobre dos pórticos metálicos, está provista de dispositivos e instalaciones combinadas, protectoras contra el sol $y$ capaces de producir el oscurecimiento necesario.

La iluminación natural se lleva a cabo a través de altas ventanas, desde los dos lados longitudinales. 


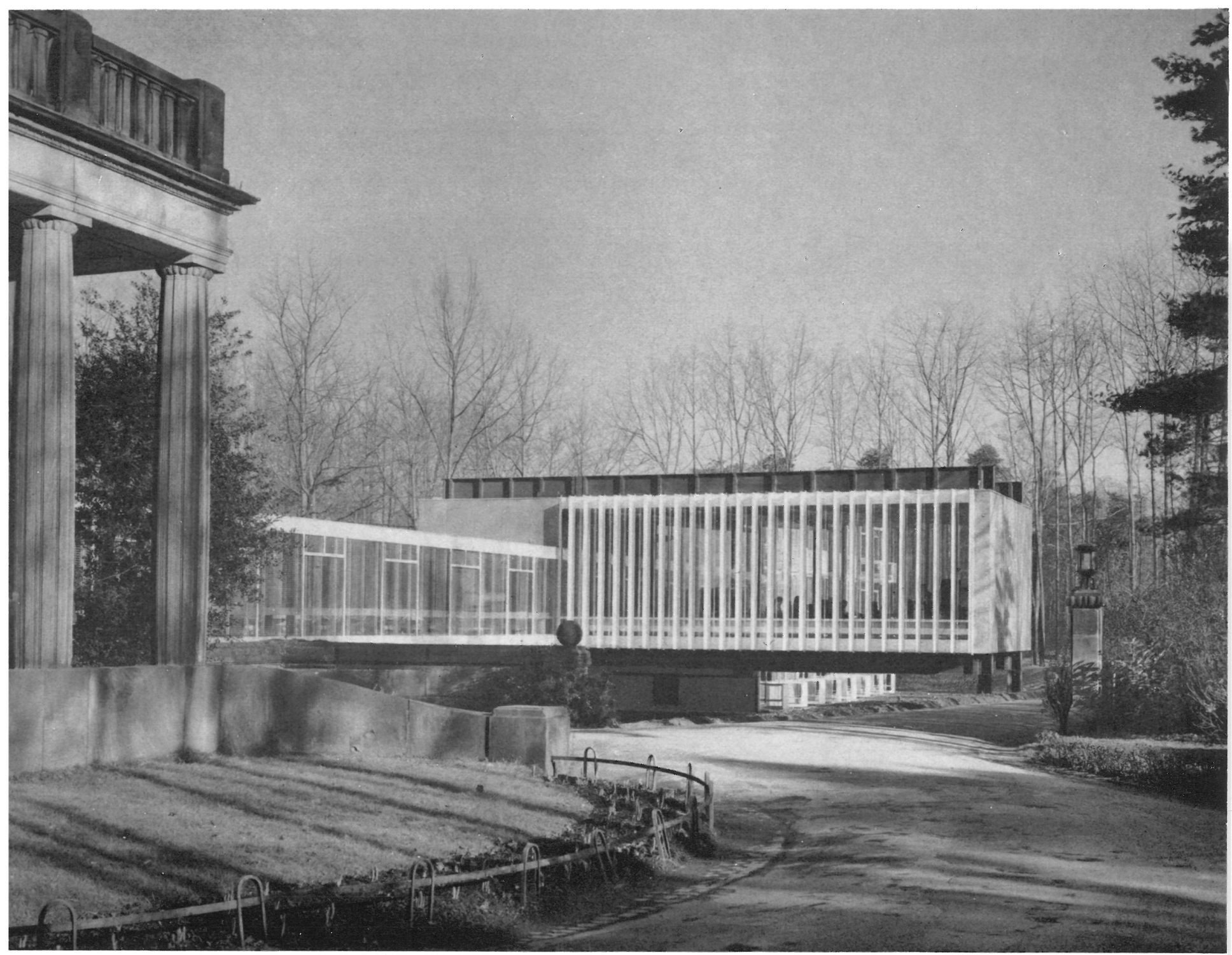

Forma parte de la Academia Evangélica de Mülhelm Ruhr una villa-castillo, de la época de la primera guerra mundial, situada en un parque-bosque muy arbolado.

La ampliación de sus instalaciones, llevada a cabo recientemente, consiste en una residencia para huéspedes, con 42 habitaciones; un auditorio, y un amplio pasillo de unión de la parte nueva con la antigua.

Este pasillo desempeña otras varias funciones: conecta, en un mismo nivel, la planta principal de la construcción primitiva con la sala de conferencias y con la planta segunda del edificio-residencia; es una galería para pasear, y sirve como lugar de relación social para que se establezcan contactos personales, entre los congresistas, durante los descansos entre conferencia y conferencia. Tiene más de $40 \mathrm{~m}$ de largo y $5 \mathrm{~m}$ de anchura, y se halla situado a nivel superior del terreno, apoyándose sobre soportes metálicos, que permiten en su parte inferior obtener una gran diafanidad. 


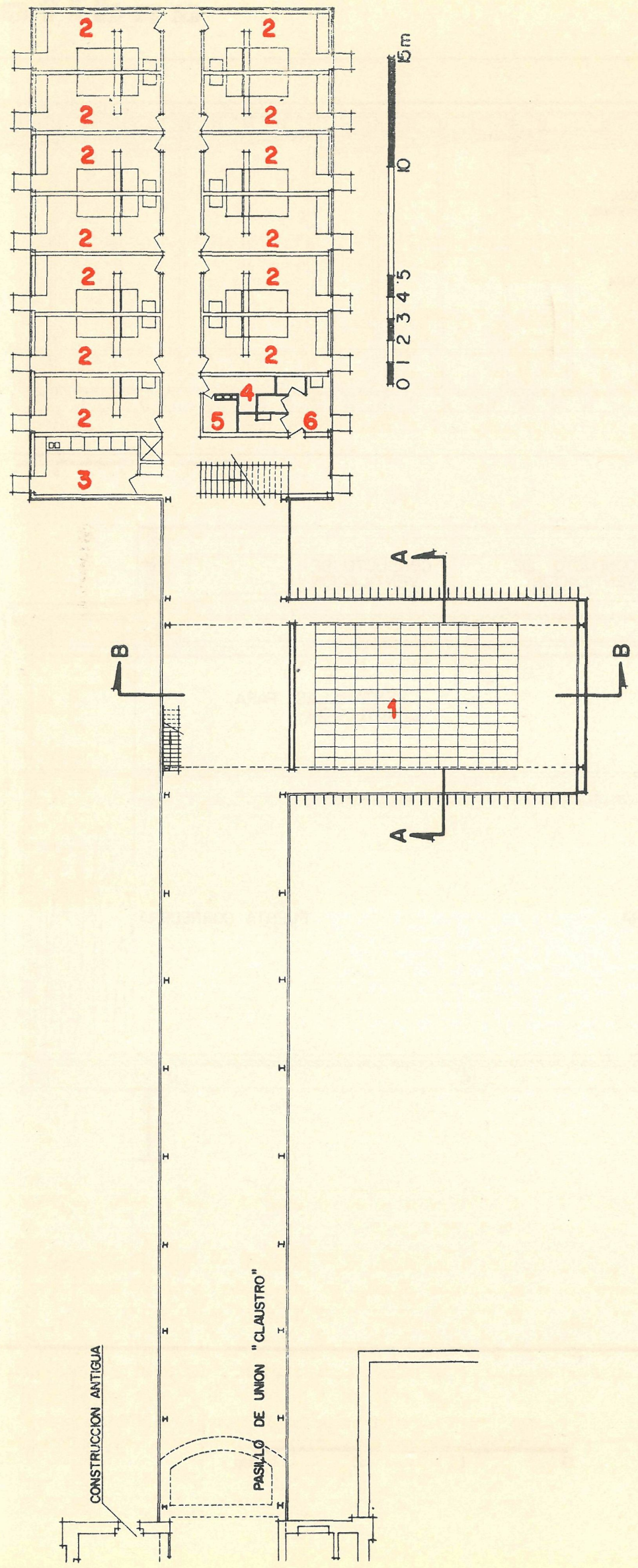

p $1 \mathbf{a} \mathbf{n}$

1. Auditorio.-2. Dormitorio.-3. Cocina de té,-4. Ducha.-5, Baño.-6. W. C.

Su cerramiento se ha realizado a base de grandes superficies acristaladas, que ayudan a dar la sensación de incorporar la naturaleza y el ambiente exterior a la parte interior, creancio así una fusión con el bello paisaje circundante. Por otra parte, su longitud proporciona la distancia necesaria entre el edificio antiguo y los nuevos.

En la construcción de sus muros exteriores, prefabricados, han sido empleados cristales Thermopane, placas de fibrocemento de conexión, con capa aislante, perfiles y chapas metálicas. Los techos nervados, de hormigón armado, se apoyan sobre I. p. n. 16.

En el edificio para huéspedes, los forjacios de hormigón armado descansan sobre la fábrica de las paredes de separación de las habitaciones.

El borde del techo presenta unas U metálicas, de las que están suspendidos los bastidores metálicos de las fachadas. Cada elemento de bastidor comprende: la superficie exterior de una habitación con ventana, antepecho y la puerta del balcón. 

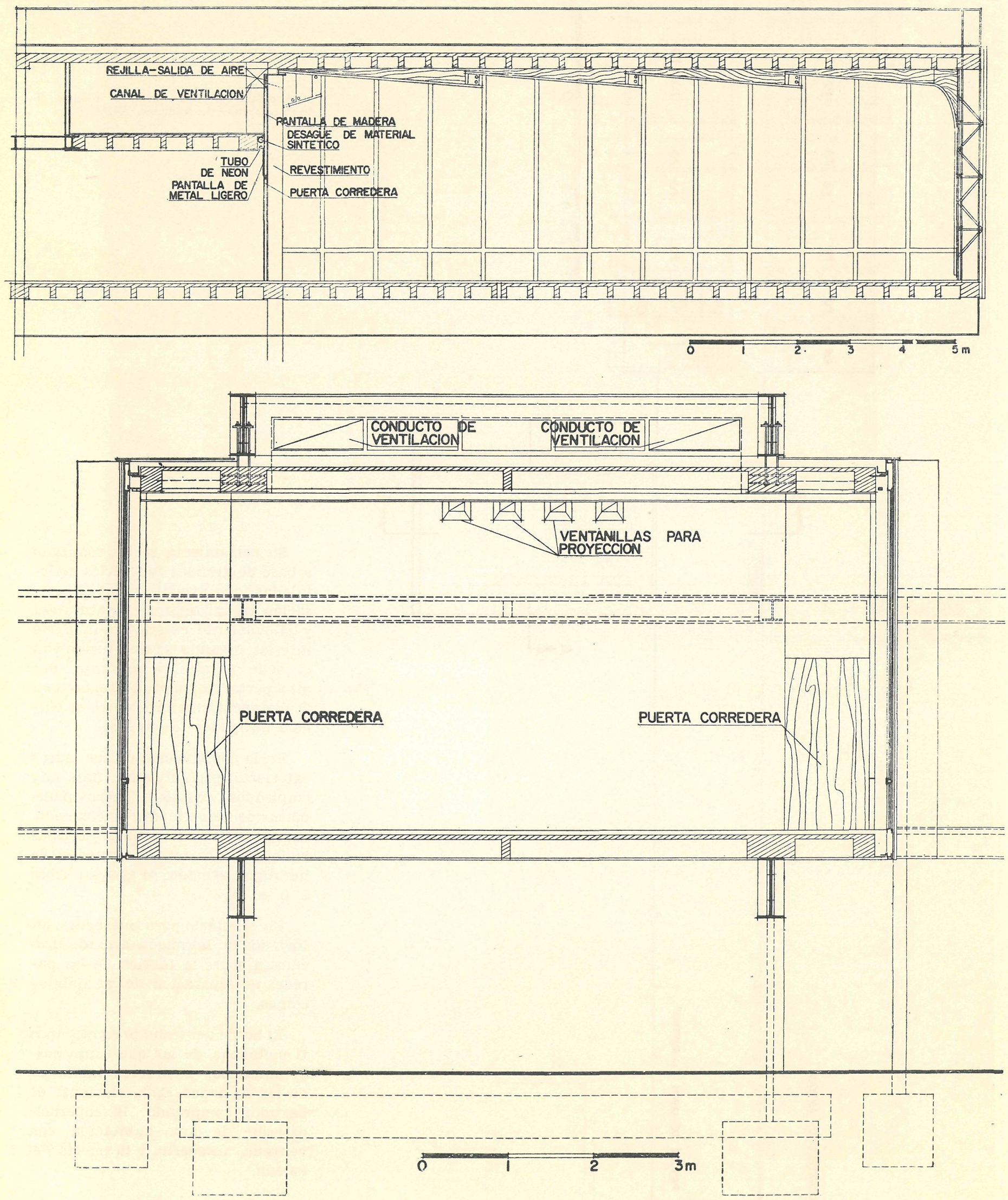

sección $4-4$ 

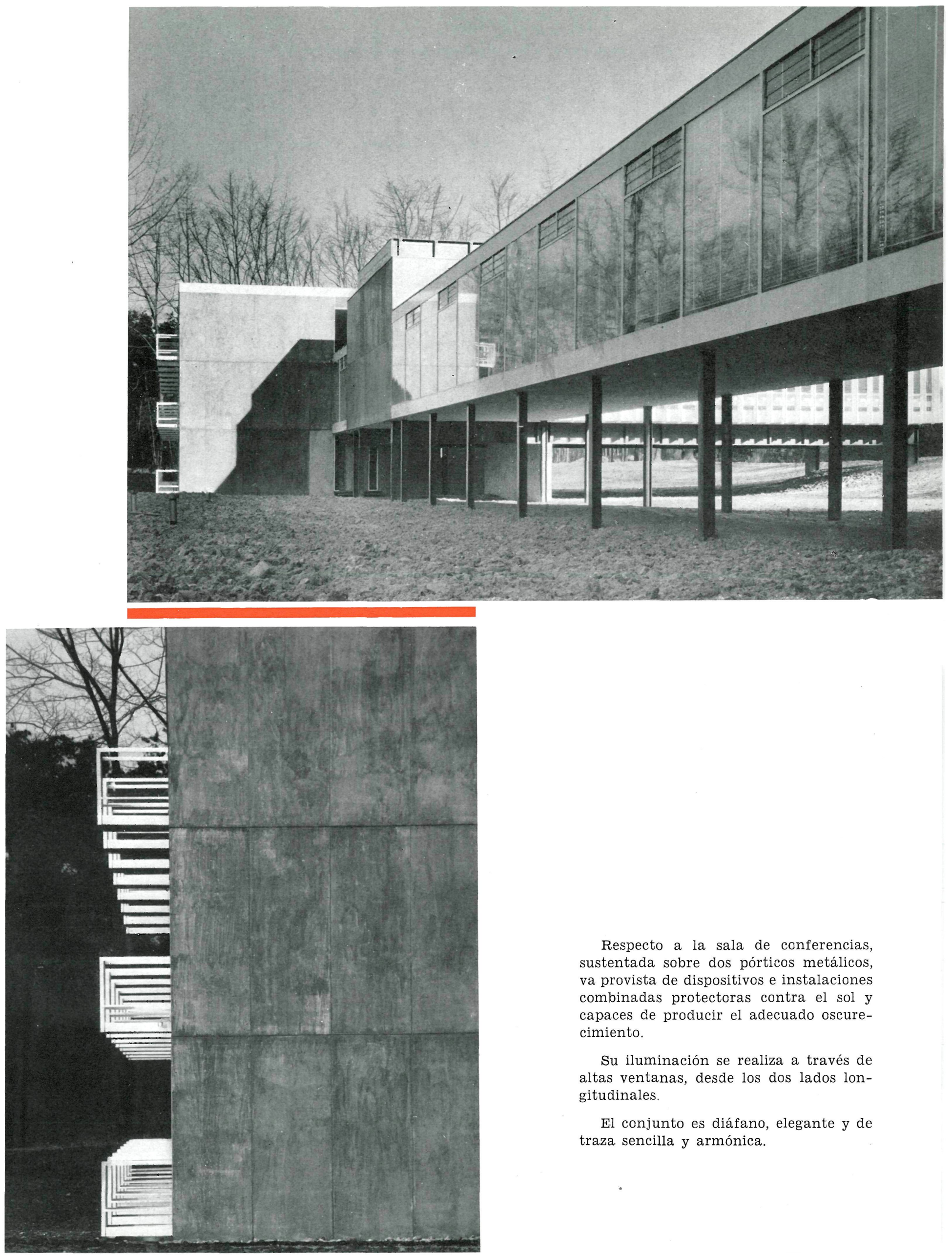

Respecto a la sala de conferencias, sustentada sobre dos pórticos metálicos, va provista de dispositivos e instalaciones combinadas protectoras contra el sol y capaces de producir el adecuado oscurecimiento.

$\mathrm{Su}$ iluminación se realiza a través de altas ventanas, desde los dos lados longitudinales.

El conjunto es diáfano, elegante y de traza sencilla y armónica. 

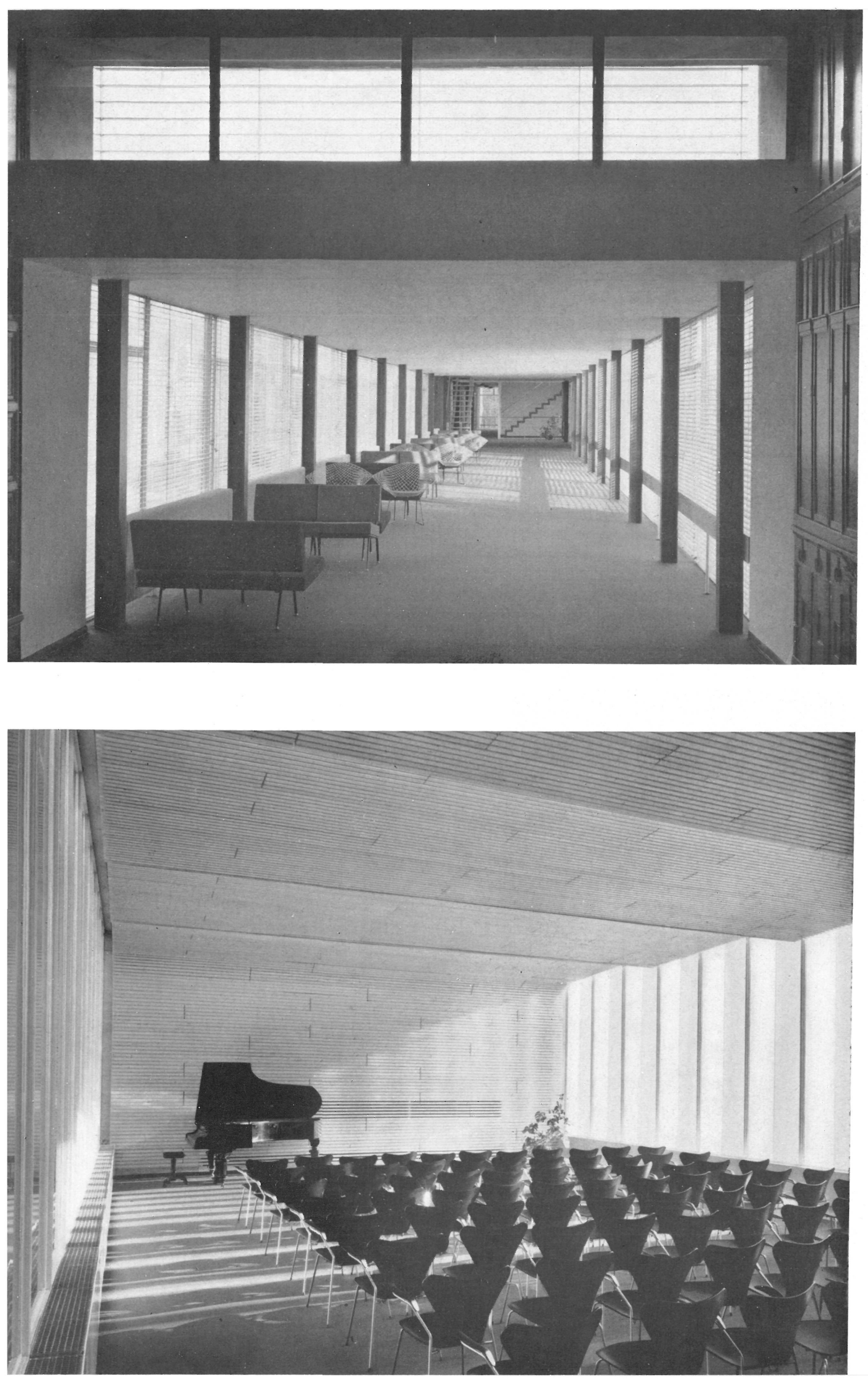

Fotos: INGE GOERTZ 


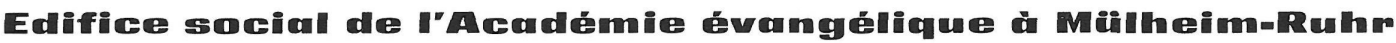

B. D. A., architectes. F. G. Winter, ingénieur diplômé.

L’agrandissement des édifices déjà existants, qui a été réalisé récemment, se compose d'une résidence comprenant quarante-deux chambres, d'un auditorium et d'un ample couloir qui unit le nouvel édifice à l'ancien.

Ce couloir remplit plusieurs fonctions: il unit, sur un même niveau, l'étage principal de la construction ancienne avec la salle de conférences et le deuxième étage de l'édifice résidentiel; c'est également une galerie pour les promenades et sert de lieu de rencontre pour les prises de contacts personnels entre congressistes, pendant les entractes des conférences.

Dans l'édifice destiné aux hôtes, les planchers en béton armé reposent sur les murs de refend des pièces.

La salle de conférences, supportée par deux portiques métalliques, est munie de dispositifs et d'installations combinées qui protègent contre le soleil et permettent l'obscurcissement nécessaire.

De hautes fenêtres facilitent l'éclairage naturel des deux côtés longitudinaux.

\section{Social building of the Envagelical Academg of Muilheim-Ruthr}

B. D. A., architects. F. G. Winter, diploma engineer.

An extension to the existing buildings was recently carried out at the above Academy. This extension comprises: a building for guests, with 42 rooms, an auditorium, and a wide passage joining the new and the old parts. This passage way has a number of uses. It connects on the same level the main floor of the existing buildings with the auditorium and with the second storey of the residential building. It constitutes a kind of promenade gallery, and it also serves as a meeting place during the intervals, for people participating in lectures and congresses.

In the guest house, the reinforced concrete flooring structure rests on the walls that separate the various rooms.

As regards the lecture hall, this is supported by two metal portal frames, and is fitted with combined installa. tions to protect it against the sun and capable of providing the necessary degree of darkness.

Natural illumination is effected by means of tall windows situated on either side of the auditorium.

\section{Gesellschartsgeböude der evangellischen Akodemie in Mirutheimerimther}

B. D. A., Architekten. F. G. Winter, Dipl.-Ing.

Die Erweiterungen der bereits bestehenden Bauten, die neuerdings durchgeführt wurden, umfassen ein Gästehaus mit 42 Zimmern, einen Hörsaal und einen geräumigen Verbindungsgang zwischen dem neuen und dem alten Flügel.

Dieser Gang hat verschiedene Aufgaben zu erfüllen: er verbindet in der gleichen Höhe das Hauptgeschoss des alten Baues mit dem Konferenzsaal und dem zweiten Stock des Wohngebäudes; er stellt einen Wandelgang dar und dient ausserdem als Ort der Fühlungnahme, um unter den Kongressteilnehmern während der Pausen zwischen den einzelnen Konferenzen persönlichen Kontakt herzustellen.

Im Gästehause ruhen die Decken aus Stahlbeton auf den Trennungswänden der Zimmer.

Der Konferenzsaal, durch zwei Stahlrahmen gestützt, ist mit schützenden Vorrichtungen und verbundenen An. lagen gegen die Sonneneinstrahlung versehen, welche auch für die nötige Verdunkelung sorgen.

Die natürliche Beleuchtung geschieht durch hohe Fenster von den beiden Längsseiten aus. 